\title{
Pharmacokinetic/Pharmacodynamic Evaluation of Dexlansoprazole Infusion Injection Compared with Lansoprazole in Healthy Chinese Adults
}

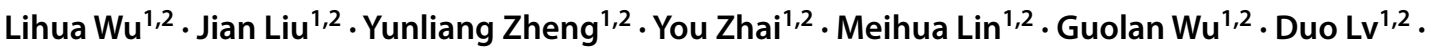 \\ Jianzhong Shentu ${ }^{1,2}$
}

Published online: 23 July 2019

(c) The Author(s) 2019

\begin{abstract}
Background and Objective This study was performed in healthy Chinese subjects to evaluate the safety and pharmacokinetic/ pharmacodynamic characteristics of a novel injection formulation of dexlansoprazole in the context of single and multiple administration, compared with the original lansoprazole injection.

Methods Helicobacter pylori-negative healthy volunteers were recruited, and 70 participants were enrolled into five dosing groups (seven males and seven females in each group), including $15 \mathrm{mg}$ once daily (qd), $15 \mathrm{mg}$ every $12 \mathrm{~h}$ (q12h), $30 \mathrm{mg} \mathrm{qd}$ and $30 \mathrm{mg} \mathrm{q} 12 \mathrm{~h}$ of dexlansoprazole treatment for 5 days, as well as $30 \mathrm{mg} \mathrm{q} 12 \mathrm{~h}$ of lansoprazole treatment for 5 days. Blood samples were collected at scheduled time spots postdose on day 1 (first dose) and day 5 (last dose). Twenty-four-hour intragastric $\mathrm{pH}$ was continuously monitored on day 0 (baseline) and days 1 and 5. Dexlansoprazole and S-lansoprazole in human plasma were determined by validated chiral liquid chromatography with tandem mass spectrometry, and the pharmacokinetic parameters were determined by a non-compartmental method using Phoenix WinNonlin software. Safety assessment included changes in vital signs and laboratory tests, physical examination findings, and incidence or reports of adverse events.

Results The half-life $\left(t_{1 / 2}\right)$ and clearance (CL) of dexlansoprazole were $1.76-2.06 \mathrm{~h}$ and $4.52-5.40 \mathrm{~L} / \mathrm{h}$, respectively, while the $t_{1 / 2}$ and CL of S-lansoprazole were $0.87-1.02 \mathrm{~h}$ and 34.66-35.98 L/h, respectively. No drug accumulation after repeated administration was noted. Administration of lansoprazole $30 \mathrm{mg}$ resulted in higher area under the concentration-time curve from time zero to the last measurable concentration $\left(\mathrm{AUC}_{\mathrm{t}}\right)$ of dexlansoprazole than that of dexlansoprazole $15 \mathrm{mg}(p=0.026)$. Zero to $24 \mathrm{~h}$ after $\mathrm{q} 12 \mathrm{~h}$ multiple dosing, median and mean intragastric $\mathrm{pH}$, percentage of time with the intragastric $\mathrm{pH}$ above $4.0[\mathrm{TpH} \geq 4.0(\%)]$ and percentage of time with the intragastric $\mathrm{pH}$ above $6.0[\mathrm{TpH} \geq 6.0(\%)]$ in the dexlansoprazole $15 \mathrm{mg}$ q12h group were $6.07 \pm 0.61,5.70 \pm 0.76,83.58 \pm 12.34$, and $53.70 \pm 17.06$, respectively, which was similar to the lansoprazole $30 \mathrm{mg}$ q12h group, i.e. $6.15 \pm 0.62,5.88 \pm 0.67,87.26 \pm 12.08$ and $57.00 \pm 16.35$, respectively. A weak positive correlation between dexlansoprazole $\mathrm{AUC}_{\mathrm{t}}$ and baseline-adjusted $\mathrm{TpH} \geq 4.0(\%)$ over 0-24 h was observed, with Pearson correlation coefficients of $0.437(p=0.029)$, while no correlation was observed between $\mathrm{AUC}_{\mathrm{t}}$ and baseline-adjusted $\mathrm{TpH}$ $\geq 6.0(\%)$ over $0-24 \mathrm{~h}$.

Conclusion Every $12 \mathrm{~h}$ intravenous dosing of dexlansoprazole up to $30 \mathrm{mg}$ for 5 days was safe and well-tolerated in healthy Chinese subjects. Every $12 \mathrm{~h}$ dosing of dexlansoprazole $15 \mathrm{mg}$ has a comparable effect of gastric acid inhibition as lansoprazole $30 \mathrm{mg} \mathrm{q} 12 \mathrm{~h}$.
\end{abstract}

Trial Registration ClinicalTrials.gov ID NCT03120273.

Lihua $\mathrm{Wu}$

wlh_xu@zju.edu.cn

$\triangle$ Jianzhong Shentu

stjz@zju.edu.cn

1 Research Center of Clinical Pharmacy, First Affliated Hospital, College of Medicine, Zhejiang University, \#79

Qingchun Road, Hangzhou 310003, China
2 Zhejiang Provincial Key Laboratory for Drug Evaluation and Clinical Research, Hangzhou, China 


\section{Key Points}

No accumulation was observed after every $12 \mathrm{~h}$ (q12h) intravenous administration of up to $30 \mathrm{mg}$ of dexlansoprazole for 5 days.

No significant differences in baseline-adjusted median and mean intragastric $\mathrm{pH}$, percentage of time with the intragastric $\mathrm{pH}$ above $4.0[\mathrm{TpH} \geq 4.0(\%)]$ and percentage of time with the intragastric $\mathrm{pH}$ above $6.0[\mathrm{TpH} \geq$ $6.0(\%)]$ were observed between dexlansoprazole $15 \mathrm{mg}$ and lansoprazole $30 \mathrm{mg}$ during any intervals.

Baseline-adjusted median and mean intragastric $\mathrm{pH}$, $\mathrm{TpH} \geq 4.0(\%)$ and $\mathrm{TpH} \geq 6.0(\%)$ for dexlansoprazole $30 \mathrm{mg}$ were significantly higher than those of dexlansoprazole $15 \mathrm{mg}$ only $0-4 \mathrm{~h}$ after the first administration, suggesting that increasing the dose from 15 to $30 \mathrm{mg}$ presented a transient better $\mathrm{pH}$ inhibition and had virtually little influence on whole-day gastric $\mathrm{pH}$ control.

\section{Introduction}

Timely endoscopic haemostatic treatment and acid suppression with high-dose intravenous proton pump inhibitors (PPIs) are cornerstones in the successful management of patients with bleeding peptic ulcers [1,2]. PPIs are supposed to inhibit gastric acid secretion by targeting the $\mathrm{H}+$, $\mathrm{K}+$-ATPase in the gastric parietal cells, and thus enhance platelet aggregation and clot formation and reduce fibrinolysis by elevated intragastric $\mathrm{pH}$ to control rebleeding from the peptic ulcer after initial endoscopic haemostasis [3, 4].

Lansoprazole, one of the six currently available PPIs in China, has been clinically used as a racemic mixture in several formulations. Due to the chiral center at the asymmetric sulfinyl group in its chemical structure, lansoprazole has two enantiomers-R-(+) and S-(-). The R-enantiomer is known as dexlansoprazole. Although the $\mathrm{R}$ and $\mathrm{S}$ isomers appeared to exhibit similar in vitro pharmacological properties, in vivo studies indicated that the gastric acid secretion inhibition activity of racemic lansoprazole was mainly from dexlansoprazole [5-7]. It showed that after a $60 \mathrm{mg}$ oral dose of racemic lansoprazole in healthy Japanese subjects, the peak concentration of the R-isomer was 2.2- to 5.8-fold higher than that of the $\mathrm{S}$-isomer, and the R/S ratios for the area under the concentration-time curve (AUC) was 5.8-12.7 [5, 6]. Following intravenous administration of racemic lansoprazole $30 \mathrm{mg}$, the mean clearance (CL) of the $\mathrm{S}$-isomer was more than fourfold higher than that observed with the R-isomer, and the mean volume of distribution $\left(V_{\mathrm{d}}\right)$ at steady-state for the $\mathrm{S}$-isomer was approximately twice that of the R-isomer [7]. Thus, after administration of lansoprazole, dexlansoprazole is the predominant isomer in the circulation as it has slower in vivo $\mathrm{CL}$ and greater systemic exposure than S-lansoprazole. Although higher plasma protein binding of dexlansoprazole, compared with the S-isomer [8], will counteract the difference seen in total plasma concentrations of the enantiomers, pharmaceutical manufacturers have been trying to develop the single enantiomer of lansoprazole in order to improve drug activity. The first formulation of dexlansoprazole, a delayed-release capsule manufactured by Takeda Pharmaceuticals America, Inc. (DEXILANT ${ }^{\circledR}$ ), was approved by the US FDA in 2009 for the healing of all grades of erosive esophagitis and for treating heartburn associated with symptomatic non-erosive gastroesophageal reflux disease (GERD) [9].

A novel injection formulation of dexlansoprazole has recently been developed for the treatment of acute peptic ulcer bleeding, or for patients with acid-related disease who are unable to swallow medications. A previous study to evaluate the safety and tolerability of dexlansoprazole injection after a single intravenous administration in healthy adults was carried out at our center from February to April 2017 (unpublished data). A total of 21 subjects received an intravenous infusion of dexlansoprazole ranging from 7.5 to $90 \mathrm{mg}$, while another seven subjects received placebo, demonstrating that a single dose of dexlansoprazole injection up to $90 \mathrm{mg}$ was safe and well-tolerated. No dose-limiting toxicity (DLT) or serious adverse events (SAEs) were recorded. Subsequently, we carried out the current study to investigate the pharmacokinetic and pharmacodynamic characteristics of dexlansoprazole after single and multiple intravenous administrations in healthy Chinese adults, compared with the original lansoprazole injection.

\section{Subjects and Methods}

\subsection{Study Drugs and Administration}

The investigational dexlansoprazole injection $(15 \mathrm{mg}$ per vial) was manufactured by Jiangsu Aosaikang Pharmaceutical Co., Ltd, China (batch number 160901, expiry date August 2018), and the active comparator (lansoprazole; $30 \mathrm{mg}$ per vial) was Takepron ${ }^{\circledR}$ injection, a commercially available original preparation from Takeda Pharmaceutical Company Limited, Japan (batch number H474, expiry date November 2017). Subjects received an intravenous infusion of dexlansoprazole or lansoprazole injection in a $100 \mathrm{~mL}$ sterile saline solution during a 30-min period using an infusion pump. 


\subsection{Subjects}

Suitable Chinese male and female adults aged $>18$ years, with a body mass index ranging from 19 to $28 \mathrm{~kg} / \mathrm{m}^{2}$, were recruited as healthy volunteers at the phase I unit, Research Center of Clinical Pharmacy, First Affliated Hospital, College of Medicine, Zhejiang University, China, in accordance with the Declaration of Helsinki and the Good Clinical Practice (GCP) guideline, during June to September 2017. All volunteers provided written informed consent to participate in the study after being well-informed about the study objectives, procedures, and possible risks. Subjects were determined to be in good health based on medical history, vital signs, physical examination, laboratory tests, 12-lead electrocardiography (ECG) and chest x-ray. Female participants of childbearing potential must have had a negative serum pregnancy test at screening, and agreed to use routinely adequate contraception. Male participants agreed to use adequate contraception and had no plans to donate sperm. Exclusion criteria were a positive Helicobacter pylori infection test; a history of known allergy or sensitivity to any drugs; a history of any clinically significant diseases, including (but not limited to) cardiovascular, respiratory, hepatic, renal, or gastrointestinal diseases; a history of alcohol or drug abuse; heavy smokers; participation in another study with an investigational drug within the last 3 months preceding this study; blood donation within the last 2 months $(\geq 400 \mathrm{~mL}$ ), or have a plan to donate blood within 1 month after completion of this study; and intake of any other drug that might influence the results of the trial during 2 weeks previous to the start of this study. Additional inclusion and exclusion criteria are detailed at ClinicalTrials.gov (https:// www.clinicaltrials.gov; NCT03120273).

\subsection{Study Design and Treatment}

This was a single center, open-label, single- and multipledose pharmacokinetic/pharmacodynamic study. The protocol was reviewed and approved by the Ethics Committee of the First Affiliated Hospital, College of Medicine, Zhejiang University (approval number 2016-EC-81).

Five dosing groups were designed, i.e. $15 \mathrm{mg}$ once daily (qd; group A), $15 \mathrm{mg}$ every $12 \mathrm{~h}$ (q12h; group B), $30 \mathrm{mg} \mathrm{qd}$ (group C) and $30 \mathrm{mg} \mathrm{q} 12 \mathrm{~h}$ (group D) in the dexlansoprazole treatment arm for 5 days, and $30 \mathrm{mg} \mathrm{q12h}$ (group E) in an active comparator (lansoprazole) treatment arm for 5 days. Seventy volunteers were enrolled in this study, with 14 subjects in each group. All volunteers were confined to the phase I unit, beginning the evening before baseline intubation. On day 0 , subjects received $\mathrm{pH}$ catheter placement for baseline 24-h intragastric $\mathrm{pH}$ monitoring. On day 1, after fasting for $10 \mathrm{~h}$, a single intravenous dose of dexlansoprazole or lansoprazole was administered, and all subjects were sampled for pharmacokinetic analysis. Meanwhile, intragastric $\mathrm{pH}$ was continuously monitored for another $24 \mathrm{~h}$. Subsequently, all subjects proceeded to multiple dosing as scheduled. On day 5, subjects received intubation transnasally, again for 24-h intragastric $\mathrm{pH}$ monitoring, and were sampled for pharmacokinetic analysis after the last morning dose. The volunteers were ambulatory, were prohibited from strenuous activity, smoking, alcohol, grapefruit juice and caffeine consumption, and were closely monitored by qualified staff throughout the entire confinement period of intubation and blood sampling. Water intake was strictly controlled on days 0,1 and 5 , and a standardized lunch and dinner were provided at 4 and $10 \mathrm{~h}$ postdosing on days 0,1 and 5 .

\subsection{Blood Sampling and Analytical Determinations}

A series of venous blood samples $(4 \mathrm{~mL})$ were collected in heparinized tubes at 0 (predose), 15, 30, and $45 \mathrm{~min}$, and $1,1.5,2,3,4,5,6,8,10,12 \mathrm{~h}$ postdose on day 1 (first dose) and day 5 (last morning dose). Samples were centrifuged immediately at $4{ }^{\circ} \mathrm{C}, 3500 \mathrm{rpm}$ for $10 \mathrm{~min}$, and plasma was separated and stored at $-70 \pm 10{ }^{\circ} \mathrm{C}$ until analysis. All blood samples were light-proof, from collecting to processing and storage.

An enantioselective method was developed and validated for the simultaneous determination of dexlansoprazole and S-lansoprazole in human plasma by chiral liquid chromatography with tandem mass spectrometry [10]. This method was applied to the present pharmacokinetic study. Specifically, lansoprazole enantiomers and internal standard (d4-lansoprazole) were extracted from plasma using acetonitrile as the protein-precipitating agent. Baseline chiral separation was achieved within $8.0 \mathrm{~min}$ on a Chiralpak IC column $(150 \mathrm{~mm} \times 4.6 \mathrm{~mm}, 5 \mu \mathrm{m})$ with a column temperature of $30^{\circ} \mathrm{C}$. The mobile phase consisted of $10 \mathrm{mM}$ ammonium acetate solution containing $0.05 \%$ acetic acid/acetonitrile (50:50, v/v). Mass spectrometric analysis was performed using an API 4000 mass spectrometer coupled with an electrospray ionization source in positive ion mode. Multiple reaction monitoring transitions of $\mathrm{m} / \mathrm{z} 370.1 \rightarrow 252.0$ and $374.1 \rightarrow 252.0$ were used to quantify lansoprazole enantiomers and d4-lansoprazole, respectively. For each enantiomer, no apparent matrix effect was found, the calibration curve was linear over $5.00-4000 \mathrm{ng} / \mathrm{mL}$, the intra- and interday precisions were below $8.0 \%$, and the accuracy was -2.9 to $10.05 \%$.

\subsection{Pharmacodynamic Measurement}

To assess the pharmadynamic effect, an ambulatory $\mathrm{pH}$ measurement system (Orion II; Medical Measurement 
Systems B.V., Enschede, The Netherlands) with a disposable pH catheter (pHersaflex; Medical Measurement Systems B.V., Enschede, The Netherlands) was used to monitor intragastric $\mathrm{pH}$. A baseline $\mathrm{pH}$ profile was obtained prior to intravenous administration of dexlansoprazole or lansoprazole on day 0 , followed by intragastric $\mathrm{pH}$ monitoring on day 1 (first dose) and day 5 . The intragastric $\mathrm{pH}$ data were recorded continuously for $24 \mathrm{~h}$ at baseline and for $24 \mathrm{~h}$ after a single dose and the last morning dose. Data were stored in the MMS Database (including the Measurement and Analysis Program, MMS, The Netherlands) for later analysis.

\subsection{Safety Assessment}

Safety assessment, including changes in vital signs and laboratory tests, physical examination findings, and incidence or reports of adverse events (AEs), were performed as scheduled in the protocol. Any AEs occurring during the study were collected by physicians from direct observation, spontaneous reports by volunteers, medical conditions and nonspecific inquiry, and closely followed to satisfactory resolution. The severity of AEs was evaluated according to the Common Terminology Criteria for Adverse Events (CTCAE) version 4.0, and the relationship to study treatment (possibly related, not possibly related) was also assessed.

\subsection{Pharmacokinetic/Pharmacodynamic and Statistical Analysis}

The maximum measured concentration over the sampling period $\left(C_{\max }\right)$ was obtained directly from the observed data. The pharmacokinetic parameters AUC from time zero to infinity $\left(\mathrm{AUC}_{\infty}\right)$, $\mathrm{AUC}$ from time zero to the last measurable concentration $\left(\mathrm{AUC}_{\mathrm{t}}\right)$, total $\mathrm{CL}, V_{\mathrm{d}}$, and elimination half-life $\left(t_{1 / 2}\right)$ were determined using a noncompartmental method with Phoenix WinNonlin software, version 6.3 (Pharsight Corporation, Sunnyvale, CA, USA). Pharmacodynamic parameters, including median $\mathrm{pH}$, mean $\mathrm{pH}$, percentage of time with the intragastric $\mathrm{pH}$ above $4.0[\mathrm{TpH} \geq$ $4.0(\%)$ ], percentage of time with the intragastric $\mathrm{pH}$ above $6.0[\mathrm{TpH} \geq 6.0(\%)]$, and baseline-adjusted intragastric $\mathrm{pH}$ parameters were calculated using the MMS software, and compared according to the time intervals of $0-24,0-4,0-12$, and $12-24 \mathrm{~h}$ postdosing. Statistical analysis was performed using SAS ${ }^{\circledR}$ version 9.4 software (SAS Institute, Cary, NC, USA). One-way analysis of variance (ANOVA) or the Wilcoxon rank-sum test after a normality test were used to assess the differences, and a $p$ value $<0.05$ was considered statistically significant. Pearson's correlation coefficient was calculated to assess the pharmacokinetic/pharmacodynamic relationship.

\section{Results}

\subsection{Subject Baseline Characteristics}

As shown in Fig. 1, a total of 262 healthy Chinese adult volunteers were screened from June to September 2017. Ultimately, 70 participants were enrolled, with seven males and seven females in each group. Table 1 summarizes the demographic characteristics of the subjects. No statistically significant differences in baseline demographics were found among the groups.

\subsection{Pharmacokinetic Properties}

All subjects completed the whole study and were included in the pharmacokinetic analyses. For single-dose pharmacokinetic evaluation, we combined groups $\mathrm{A}$ and $\mathrm{B}$ as a singledose group of dexlansoprazole $15 \mathrm{mg}$, while groups $\mathrm{C}$ and $\mathrm{D}$ were combined into another single-dose group of dexlansoprazole $30 \mathrm{mg}$. The mean plasma concentration-time profiles of dexlansoprazole obtained after single intravenous administration of dexlansoprazole 15 or $30 \mathrm{mg}$ and lansoprazole $30 \mathrm{mg}$ are shown in Fig. 2. The major pharmacokinetic parameters of dexlansoprazole are summarized in Table 2. An increase in dexlansoprazole dose from 15 to $30 \mathrm{mg}$ resulted in a dose-proportional increase in $C_{\max }$ and AUC following a single intravenous administration. The $t_{1 / 2}, \mathrm{CL}$ and $V_{\mathrm{d}}$ parameters were independent of dose. No S-lansoprazole was detectable in plasma following intravenous administration of dexlansoprazole, therefore racemic conversion of dexlansoprazole to S-lansoprazole did not occur. After intravenous infusion of lansoprazole, dexlansoprazole was the major isomer in the plasma, while S-lansoprazole displayed a rapid CL, with $t_{1 / 2}$ being approximately $1 \mathrm{~h}$. Compared with dexlansoprazole $15 \mathrm{mg}$, administration of lansoprazole $30 \mathrm{mg}$ resulted in a statistically significant increase in both $\operatorname{AUC}_{\mathrm{t}}(p=0.026)$ and $\mathrm{AUC}_{\infty}(p=0.037)$ of dexlansoprazole. After multiple intravenous doses, the $C_{\max }$ and AUC parameters remained similar to those of the single dose on day 1 , indicating that dexlansoprazole was not accumulating after repeated administration. The major pharmacokinetic parameters after repeated administration are summarized in Table 3. Consistent with the results from a single dose, $\mathrm{AUC}_{\mathrm{t}}$ and $V_{\mathrm{d}}$ of dexlansoprazole in the lansoprazole $30 \mathrm{mg}$ group were also significantly higher than in the dexlansoprazole $15 \mathrm{mg}$ group with repeated administration.

\subsection{Pharmacodynamic Properties}

As shown in Tables 4 and 5, the baseline median and mean intragastric $\mathrm{pH}$ was $1.44-1.91$ and $1.76-2.37$, respectively. 


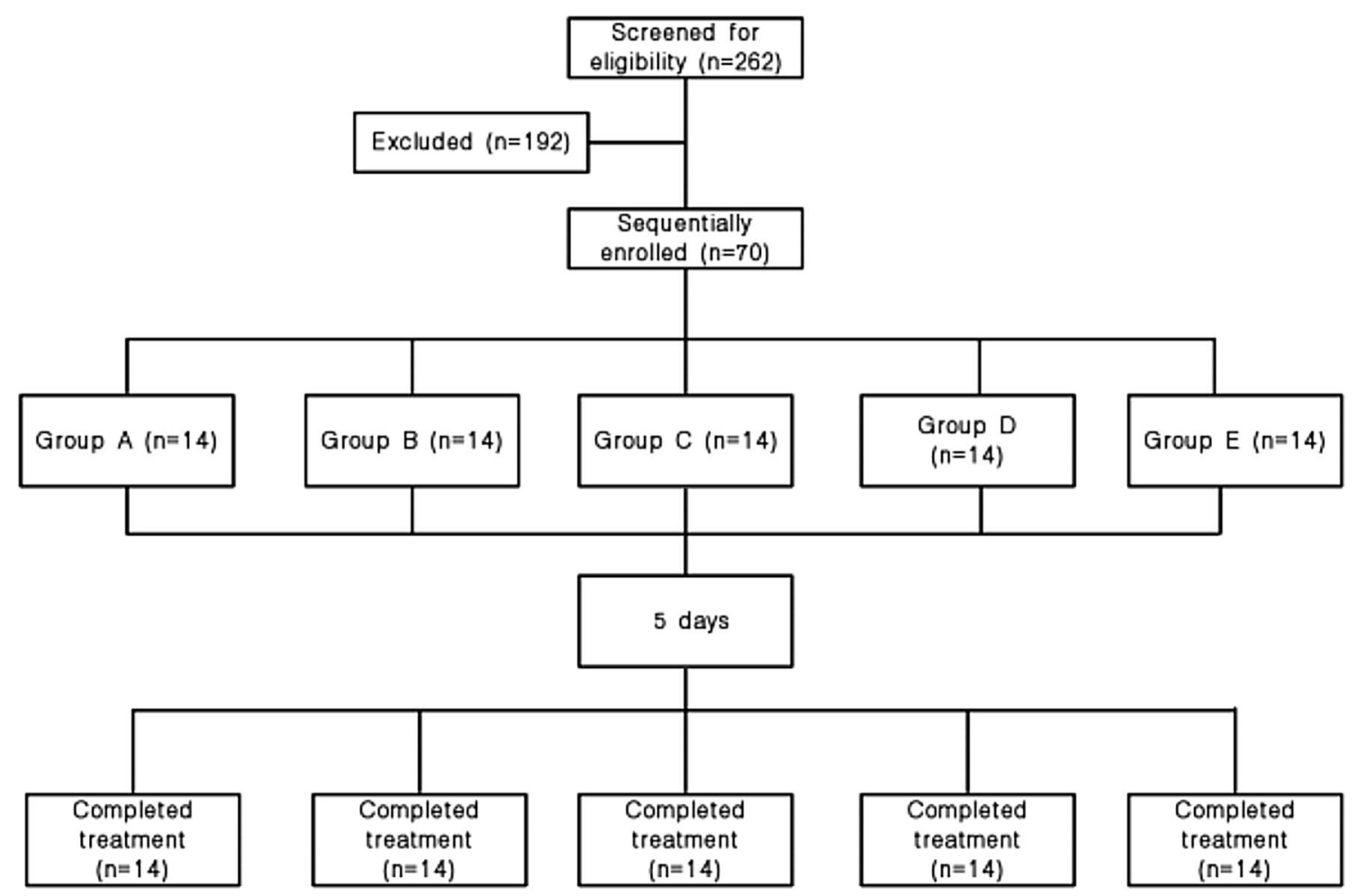

Fig. 1 Study screening, enrollment, dosing and follow-up. All 70 participants completed the study regimen

Table 1 Demographic characteristics of the study subjects $[n=70]$

\begin{tabular}{|c|c|c|c|c|c|}
\hline \multirow[t]{2}{*}{ Characteristic } & \multicolumn{4}{|c|}{ Dexlansoprazole group } & \multirow{2}{*}{$\begin{array}{l}\text { Lansoprazole group } \\
\text { E (30 mg q12h) }\end{array}$} \\
\hline & $\mathrm{A}(15 \mathrm{mg} \mathrm{qd})$ & B (15 mg q12h) & $\mathrm{C}(30 \mathrm{mg}$ qd $)$ & $\mathrm{D}(30 \mathrm{mg} \mathrm{q} 12 \mathrm{~h})$ & \\
\hline No. of subjects & 14 & 14 & 14 & 14 & 14 \\
\hline \multicolumn{6}{|l|}{ Age, years } \\
\hline Mean (SD) & $29.1(8.51)$ & $26.9(4.66)$ & $25.3(4.25)$ & $27.9(5.04)$ & $29.6(6.10)$ \\
\hline Minimum-maximum & $19-45$ & $19-36$ & $19-33$ & $20-40$ & $19-43$ \\
\hline \multicolumn{6}{|l|}{ Weight, kg } \\
\hline Mean (SD) & $63.2(11.14)$ & $61.4(7.84)$ & $58.6(6.74)$ & $59.8(8.38)$ & $57.7(6.90)$ \\
\hline Minimum-maximum & $46.3-78.2$ & $47.6-75.3$ & $48.7-72.2$ & $48.8-81.5$ & $45.3-68.5$ \\
\hline \multicolumn{6}{|l|}{ Height, cm } \\
\hline Mean (SD) & $165.2(9.05)$ & $167.9(5.40)$ & $164.0(3.98)$ & $165.1(6.94)$ & $161.4(7.15)$ \\
\hline Minimum-maximum & $150.0-180.0$ & $157.0-176.0$ & $156.0-170.0$ & $151.0-175.0$ & $146.0-177.0$ \\
\hline \multicolumn{6}{|l|}{ BMI, $\mathrm{kg} / \mathrm{m}^{2}$} \\
\hline Mean (SD) & $22.9(2.08)$ & $21.7(2.21)$ & $21.7(1.81)$ & $21.8(1.88)$ & $22.1(1.74)$ \\
\hline Minimum-maximum & $19.8-25.8$ & $19.1-25.0$ & $19.0-25.2$ & $19.5-26.6$ & $20.3-25.2$ \\
\hline
\end{tabular}

$B M I$ body mass index, $S D$ standard deviation, $q d$ once daily, $q 12 \mathrm{~h}$ every $12 \mathrm{~h}$

No statistically significant differences in baseline intragastric $\mathrm{pH}$ were found among the groups. After intravenous administration of dexlansoprazole or lansoprazole, intragastric $\mathrm{pH}$ was significantly elevated in subjects across all groups. Changes in percentage time with the intragastric $\mathrm{pH} \geq 4.0$ or $\mathrm{pH} \geq 6.0, \mathrm{TpH} \geq 4.0(\%)$ or $\mathrm{TpH} \geq 6.0(\%)$, after intravenous administration of dexlansoprazole or lansoprazole, are shown in Tables 6 and 7.

During a $0-12 \mathrm{~h}$ interval, a single dose of dexlansoprazole $15 \mathrm{mg}$ (group B) and $30 \mathrm{mg}$ (group D) resulted in a median intragastric $\mathrm{pH}$ of $5.72 \pm 1.24$ and $6.28 \pm 0.93$, mean intragastric $\mathrm{pH}$ of $5.36 \pm 0.91$ and $5.91 \pm 0.85, \mathrm{TpH}$ 
(a)

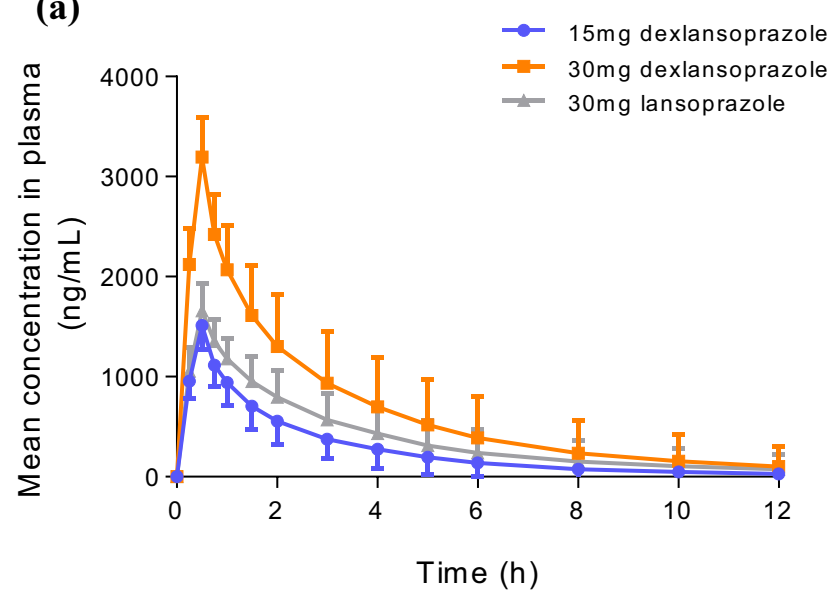

(b)

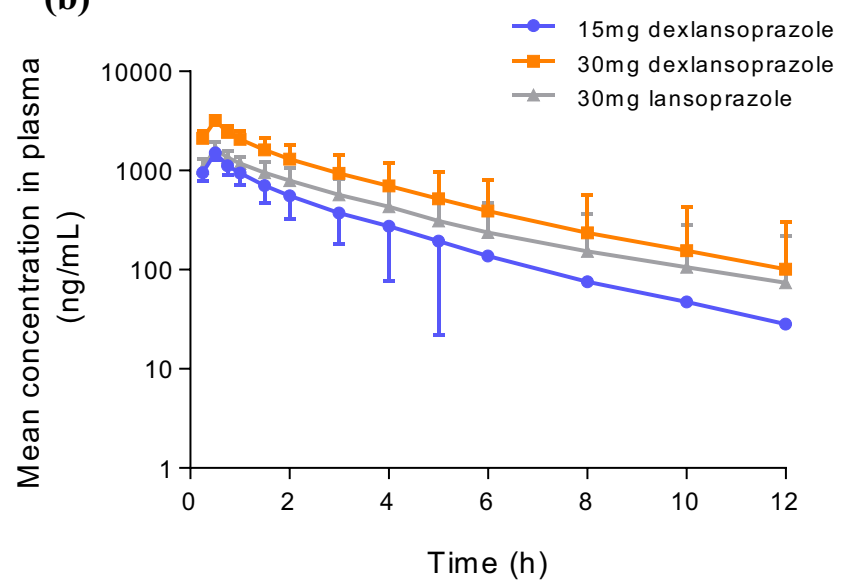

Fig. 2 Mean plasma concentration-time profiles after single intravenous administration of dexlansoprazole 15 or $30 \mathrm{mg}$ and lansoprazole $30 \mathrm{mg}$. a Linear scale; b semi-logarithmic scale

Table 2 Pharmacokinetic parameters after single intravenous administration of dexlansoprazole 15 or $30 \mathrm{mg}$ and lansoprazole $30 \mathrm{mg}$ $($ mean $\pm \mathrm{SD})$

\begin{tabular}{|c|c|c|c|c|c|c|c|c|}
\hline \multirow{3}{*}{$\begin{array}{l}\text { Param- } \\
\text { eter }\end{array}$} & \multicolumn{6}{|l|}{ Dexlansoprazole group } & \multirow{2}{*}{\multicolumn{2}{|c|}{$\begin{array}{l}\text { Lansoprazole group } \\
\text { E [N=14] }\end{array}$}} \\
\hline & \multirow[t]{2}{*}{$\mathrm{A}[N=14]$} & \multirow[t]{2}{*}{$\mathrm{B}[N=14]$} & \multirow[t]{2}{*}{$15 \mathrm{mg}[N=28]$} & \multirow[t]{2}{*}{$\mathrm{C}[N=14]$} & \multirow[t]{2}{*}{$\mathrm{D}[N=14]$} & \multirow[t]{2}{*}{$30 \mathrm{mg}[N=28]$} & & \\
\hline & & & & & & & Dexlansoprazole & S-lansoprazole \\
\hline $\begin{array}{c}C_{\max }, \\
\mathrm{ng} / \\
\mathrm{mL}\end{array}$ & $1534.86 \pm 271.92$ & $1490.00 \pm 201.45$ & $1512.43 \pm 235.93$ & $3242.14 \pm 485.27$ & $3145.64 \pm 296.21$ & $3193.89 \pm 397.55$ & $1663.50 \pm 264.11$ & $848.13 \pm 254.13$ \\
\hline$t_{1 / 2}, \mathrm{~h}$ & $2.00 \pm 1.29$ & $1.52 \pm 0.64$ & $1.76 \pm 1.03$ & $2.07 \pm 1.52$ & $2.04 \pm 1.28$ & $2.06 \pm 1.38$ & $2.32 \pm 1.74$ & $1.02 \pm 0.62$ \\
\hline $\mathrm{MRT}_{\mathrm{t}}$ & $2.28 \pm 0.97$ & $1.88 \pm 0.73$ & $2.08 \pm 1.03$ & $2.30 \pm 1.02$ & $2.34 \pm 0.92$ & $2.32 \pm 0.96$ & $2.50 \pm 1.02$ & $1.02 \pm 0.64$ \\
\hline $\begin{array}{l}\mathrm{AUC}_{\mathrm{t}} \\
\mathrm{ng} \cdot \mathrm{h} / \\
\mathrm{mL}\end{array}$ & $3706.33 \pm 1842.63$ & $2956.61 \pm 1259.20$ & $3331.47 \pm 1594.97$ & $78504.36 \pm 5251.32$ & $7705.40 \pm 3352.63$ & $8104.88 \pm 4342.22$ & $\begin{array}{l}2487.40 \pm 2461.01^{\mathrm{a}} \\
(p=0.026)\end{array}$ & $1140.25 \pm 829.56$ \\
\hline $\begin{array}{c}\mathrm{AUC}_{\infty}, \\
\mathrm{ng} \cdot \mathrm{h} / \\
\mathrm{mL}\end{array}$ & $3973.77 \pm 2373.66$ & $3022.23 \pm 1363.57$ & $3498.00 \pm 1960.30$ & $9380.59 \pm 7219.50$ & $8206.10 \pm 4318.42$ & $8793.35 \pm 5867.88$ & $\begin{array}{l}5372.52 \pm 3709.23^{\mathrm{a}} \\
(p=0.037)\end{array}$ & $1161.07 \pm 848.87$ \\
\hline$V_{\mathrm{d}}, \mathrm{L}$ & $11.00 \pm 1.51$ & $11.31 \pm 1.89$ & $11.16 \pm 1.69$ & $10.03 \pm 1.55$ & $10.57 \pm 1.28$ & $10.30 \pm 1.42$ & $\begin{array}{l}18.64 \pm 2.97^{\mathrm{a}} \\
(p=0.000)\end{array}$ & $43.65 \pm 24.30$ \\
\hline $\mathrm{CL}, \mathrm{L} / \mathrm{h}$ & $4.90 \pm 2.36$ & $5.90 \pm 2.43$ & $5.40 \pm 2.41$ & $4.65 \pm 2.62$ & $4.39 \pm 1.63$ & $4.52 \pm 2.14$ & $\begin{array}{l}7.49 \pm 3.57^{\mathrm{a}} \\
(p=0.030)\end{array}$ & $34.66 \pm 15.36$ \\
\hline
\end{tabular}

Data are expressed as arithmetic mean $\pm \mathrm{SD}$

For single-dose pharmacokinetic evaluation, we combined groups A and B as a single-dose group of dexlansoprazole $15 \mathrm{mg}(\mathrm{N}=28)$, while groups $\mathrm{C}$ and $\mathrm{D}$ were combined as a single-dose group of dexlansoprazole $30 \mathrm{mg}(N=28)$

$S D$ standard deviation, $C_{\max }$ maximum measured concentration over the sampling period, $t_{1 / 2}$ apparent elimination half-life, $M R T_{t}$ mean retention time, $A U C_{t}$ area under the concentration-time curve from time zero to the last measurable concentration, $A U C_{\infty}$ area under the concentrationtime curve from time zero to infinity, $V_{d}$ volume of distribution, $C L$ total clearance

${ }^{\mathrm{a}}$ Compared with single-dose dexlansoprazole $15 \mathrm{mg}$

$\geq 4.0(\%)$ of $71.26 \pm 15.79$ and $82.36 \pm 13.14$, and $\mathrm{TpH} \geq$ $6.0(\%)$ of $49.68 \pm 19.74$ and $59.89 \pm 19.83$, respectively. In the lansoprazole $30 \mathrm{mg}$ group (group $\mathrm{E}$ ), median and mean intragastric $\mathrm{pH}, \mathrm{TpH} \geq 4.0(\%)$ and $\mathrm{TpH} \geq 6.0(\%)$ $0-12 \mathrm{~h}$ after the first dose were $5.99 \pm 1.04,5.54 \pm 0.68$, $73.81 \pm 12.68$, and $53.36 \pm 12.95$, respectively. There were no statistically significant differences in pharmacodynamic parameters $0-12 \mathrm{~h}$ after a single dose among these three groups.

After repeated administration, all pharmacodynamic parameters showed an increase from the first dose on day 1 to the last morning dose on day 5 , which indicated further gastric acid suppression. The mean intragastric $\mathrm{pH} 0-12 \mathrm{~h}$ after the last morning dose of dexlansoprazole $15 \mathrm{mg}$ (group 
Table 3 Pharmacokinetic parameters after repeated intravenous administration of dexlansoprazole 15 or $30 \mathrm{mg}$ and lansoprazole $30 \mathrm{mg}$ $($ mean $\pm \mathrm{SD})$

\begin{tabular}{|c|c|c|c|c|c|c|}
\hline \multirow[t]{3}{*}{ Parameter } & \multicolumn{4}{|c|}{ Dexlansoprazole group } & \multirow{2}{*}{\multicolumn{2}{|c|}{$\begin{array}{l}\text { Lansoprazole group } \\
\text { E (30 mg q12h) }\end{array}$}} \\
\hline & \multirow[t]{2}{*}{ A (15 mg qd) } & \multirow[t]{2}{*}{ B (15 mg q12h) } & \multirow[t]{2}{*}{$\mathrm{C}(30 \mathrm{mg} \mathrm{qd})$} & \multirow[t]{2}{*}{ D (30 mg q12h) } & & \\
\hline & & & & & Dexlansoprazole & S-lansoprazole \\
\hline$C_{\max }, \mathrm{ng} / \mathrm{mL}$ & $1583.50 \pm 234.17$ & $1700.36 \pm 696.32$ & $3518.64 \pm 1245.55$ & $3068.29 \pm 258.06$ & $1792.43 \pm 326.01$ & $811.63 \pm 181.98$ \\
\hline$t_{1 / 2}, \mathrm{~h}$ & $2.13 \pm 1.63$ & $1.66 \pm 0.92$ & $2.06 \pm 1.30$ & $2.07 \pm 1.27$ & $2.43 \pm 1.69$ & $0.87 \pm 0.58$ \\
\hline $\mathrm{MRT}_{\mathrm{t}}$ & $2.88 \pm 2.17$ & $2.15 \pm 1.21$ & $2.84 \pm 1.97$ & $2.87 \pm 1.90$ & $3.42 \pm 2.49$ & $1.11 \pm 0.76$ \\
\hline $\begin{array}{l}\mathrm{AUC}_{\mathrm{t}}, \mathrm{ng} \cdot \mathrm{h} / \\
\mathrm{mL}\end{array}$ & $3860.13 \pm 1964.93$ & $3228.494 \pm 1935.45$ & $8878.77 \pm 5518.35$ & $8389.95 \pm 4581.11$ & $\begin{array}{l}5845.45 \pm 3916.79^{\mathrm{a}} \\
(p=0.034)\end{array}$ & $1165.52 \pm 920.46$ \\
\hline$\underset{\mathrm{mL}}{\mathrm{AUC}_{\infty}, \mathrm{ng} \cdot \mathrm{h} /}$ & $4202.46 \pm 2697.97$ & $3370.36 \pm 2285.62$ & $9690.00 \pm 7358.78$ & $9071.59 \pm 6009.33$ & $6690.33 \pm 5708.48$ & $1180.29 \pm 942.84$ \\
\hline$V_{\mathrm{d}}, \mathrm{L}$ & $10.19 \pm 1.47$ & $10.26 \pm 1.34$ & $9.25 \pm 1.57$ & $9.88 \pm 1.18$ & $\begin{array}{l}17.41 \pm 2.20^{\mathrm{a}} \\
(p=0.000)\end{array}$ & $30.55 \pm 6.17$ \\
\hline $\mathrm{CL}, \mathrm{L} / \mathrm{h}$ & $4.72 \pm 2.20$ & $5.9 \pm 2.64$ & $4.42 \pm 2.41$ & $4.37 \pm 1.77$ & $7.02 \pm 3.69$ & $35.98 \pm 17.51$ \\
\hline $\begin{array}{l}\text { Accumulation } \\
\text { index }\end{array}$ & $1.01 \pm 0.03$ & $1.02 \pm 0.05$ & $1.01 \pm 0.01$ & $1.04 \pm 0.08$ & $1.06 \pm 0.12$ & $1.00 \pm 0.01$ \\
\hline
\end{tabular}

$S D$ standard deviation, $C_{\max }$ maximum measured concentration over the sampling period, $t_{1 / 2}$ elimination half-life, $M R T_{t}$ mean retention time, $A U C_{t}$ area under the concentration-time curve from time zero to the last measurable concentration, $A U C_{\infty}$ area under the concentration-time curve from time zero to infinity, $V_{d}$ volume of distribution, $C L$ total clearance, $q d$ once daily, $q 12 \mathrm{~h}$ every $12 \mathrm{~h}$

${ }^{\mathrm{a}}$ Compared with group B (dexlansoprazole $15 \mathrm{mg} \mathrm{q} 12 \mathrm{~h}$ )

Table 4 Change in intragastric median $\mathrm{pH}$ after single or repeated intravenous administration of dexlansoprazole 15 or $30 \mathrm{mg}$ and lansoprazole $30 \mathrm{mg}($ mean $\pm \mathrm{SD})$

\begin{tabular}{|c|c|c|c|c|c|c|c|}
\hline \multirow{2}{*}{ Time period } & \multirow{2}{*}{ Variable } & \multicolumn{4}{|c|}{ Dexlansoprazole group } & \multirow{2}{*}{$\begin{array}{l}\text { Lansoprazole group } \\
\text { E (30 mg q12h) }\end{array}$} & \multirow{2}{*}{$p$ value } \\
\hline & & $\mathrm{A}(15 \mathrm{mg} \mathrm{qd})$ & B (15 mg q12h) & $\mathrm{C}(30 \mathrm{mg} \mathrm{qd})$ & $\mathrm{D}(30 \mathrm{mg}$ q12h) & & \\
\hline \multirow[t]{5}{*}{$0-24 \mathrm{~h}$} & Baseline & $1.54 \pm 0.23$ & $1.53 \pm 0.19$ & $1.57 \pm 0.18$ & $1.76 \pm 0.70$ & $1.54 \pm 0.13$ & 0.3558 \\
\hline & First dose (day 1) & $4.73 \pm 1.12$ & $5.88 \pm 0.78$ & $5.36 \pm 1.50$ & $6.15 \pm 0.85$ & $5.97 \pm 1.04$ & 0.0081 \\
\hline & Baseline-adjusted & $3.19 \pm 1.06$ & $4.35 \pm 0.75$ & $3.79 \pm 1.45$ & $4.39 \pm 0.96$ & $4.44 \pm 0.97$ & 0.0139 \\
\hline & Last dose (day 5) & $4.63 \pm 1.23$ & $6.07 \pm 0.61$ & $5.69 \pm 1.02$ & $6.34 \pm 0.45$ & $6.15 \pm 0.62$ & $<0.0001$ \\
\hline & Baseline-adjusted & $3.25 \pm 1.04$ & $4.54 \pm 0.69$ & $4.12 \pm 1.17$ & $4.56 \pm 0.92$ & $4.61 \pm 0.62$ & 0.0009 \\
\hline \multirow[t]{5}{*}{$0-12 \mathrm{~h}$} & Baseline & $1.75 \pm 0.44$ & $1.76 \pm 0.32$ & $1.61 \pm 0.33$ & $1.91 \pm 0.61$ & $1.76 \pm 0.28$ & 0.4595 \\
\hline & First dose (day 1) & $5.92 \pm 0.77$ & $5.72 \pm 1.24$ & $6.46 \pm 1.32$ & $6.28 \pm 0.93$ & $5.99 \pm 1.04$ & 0.3967 \\
\hline & Baseline-adjusted & $4.17 \pm 0.67$ & $3.96 \pm 1.28$ & $4.85 \pm 1.29$ & $4.36 \pm 0.96$ & $4.23 \pm 1.12$ & 0.2795 \\
\hline & Last dose (day 5) & $5.95 \pm 0.72$ & $6.11 \pm 0.78$ & $6.63 \pm 0.42$ & $6.50 \pm 0.33$ & $6.54 \pm 0.56$ & 0.0134 \\
\hline & Baseline-adjusted & $4.27 \pm 0.71$ & $4.35 \pm 0.79$ & $5.02 \pm 0.72$ & $4.57 \pm 0.69$ & $4.77 \pm 0.63$ & 0.0535 \\
\hline \multirow[t]{5}{*}{$12-24 \mathrm{~h}$} & Baseline & $1.45 \pm 0.19$ & $1.44 \pm 0.21$ & $1.54 \pm 0.17$ & $1.66 \pm 0.76$ & $1.46 \pm 0.14$ & 0.4992 \\
\hline & First dose (day 1) & $3.58 \pm 1.50$ & $5.89 \pm 0.80$ & $4.27 \pm 1.82$ & $6.24 \pm 1.00$ & $5.89 \pm 1.14$ & $<0.0001$ \\
\hline & Baseline-adjusted & $2.12 \pm 1.47$ & $4.45 \pm 0.73$ & $2.74 \pm 1.77$ & $4.57 \pm 1.21$ & $4.44 \pm 1.05$ & $<0.0001$ \\
\hline & Last dose (day 5) & $3.44 \pm 1.71$ & $5.83 \pm 0.65$ & $4.32 \pm 1.63$ & $6.05 \pm 0.61$ & $5.71 \pm 0.79$ & $<0.0001$ \\
\hline & Baseline-adjusted & $2.12 \pm 1.64$ & $4.39 \pm 0.65$ & $2.78 \pm 1.70$ & $4.38 \pm 1.11$ & $4.25 \pm 0.80$ & $<0.0001$ \\
\hline \multirow[t]{3}{*}{$0-4 \mathrm{~h}$} & Baseline & $1.55 \pm 0.27$ & $1.42 \pm 0.20$ & $1.71 \pm 0.73$ & $1.69 \pm 0.57$ & $1.63 \pm 0.39$ & 0.4826 \\
\hline & First dose (day 1) & $3.95 \pm 1.86$ & $4.11 \pm 1.55$ & $5.48 \pm 1.77$ & $5.24 \pm 1.73$ & $3.94 \pm 1.33$ & 0.0304 \\
\hline & Baseline-adjusted & $2.41 \pm 1.75$ & $2.69 \pm 1.51$ & $3.76 \pm 1.69$ & $3.54 \pm 1.61$ & $2.31 \pm 1.24$ & 0.0495 \\
\hline
\end{tabular}

$p$ values represent the comparison among the five groups

$S D$ standard deviation, $q d$ once daily, $q 12 h$ every $12 \mathrm{~h}$ 
Table 5 Change in intragastric mean $\mathrm{pH}$ after single or repeated intravenous administration of dexlansoprazole 15 or $30 \mathrm{mg}$ and lansoprazole $30 \mathrm{mg}($ mean $\pm \mathrm{SD})$

\begin{tabular}{|c|c|c|c|c|c|c|c|}
\hline \multirow[t]{2}{*}{ Time period } & \multirow[t]{2}{*}{ Variable } & \multicolumn{4}{|c|}{ Dexlansoprazole group } & \multirow{2}{*}{$\begin{array}{l}\text { Lansoprazole group } \\
\text { E (30 mg q12h) }\end{array}$} & \multirow[t]{2}{*}{$p$ value } \\
\hline & & A (15 mg qd) & B (15 mg q12h) & $\mathrm{C}(30 \mathrm{mg} \mathrm{qd})$ & $\mathrm{D}(30 \mathrm{mg} \mathrm{q} 12 \mathrm{~h})$ & & \\
\hline \multirow[t]{5}{*}{$0-24 \mathrm{~h}$} & Baseline & $2.07 \pm 0.46$ & $2.09 \pm 0.31$ & $2.01 \pm 0.37$ & $2.17 \pm 0.74$ & $2.03 \pm 0.25$ & 0.9031 \\
\hline & First dose (day 1) & $4.72 \pm 0.84$ & $5.54 \pm 0.73$ & $5.28 \pm 1.15$ & $6.01 \pm 0.80$ & $5.66 \pm 0.77$ & 0.0051 \\
\hline & Baseline-adjusted & $2.65 \pm 0.77$ & $3.44 \pm 0.76$ & $3.26 \pm 1.01$ & $3.84 \pm 0.87$ & $3.63 \pm 0.79$ & 0.0073 \\
\hline & Last dose (day 5) & $4.66 \pm 0.91$ & $5.70 \pm 0.76$ & $5.26 \pm 0.88$ & $6.15 \pm 0.50$ & $5.88 \pm 0.67$ & 0.0001 \\
\hline & Baseline-adjusted & $2.68 \pm 0.75$ & $3.61 \pm 0.86$ & $3.24 \pm 1.13$ & $3.96 \pm 0.89$ & $3.85 \pm 0.65$ & 0.0021 \\
\hline \multirow[t]{5}{*}{$0-12 \mathrm{~h}$} & Baseline & $2.28 \pm 0.45$ & $2.37 \pm 0.25$ & $2.14 \pm 0.44$ & $2.34 \pm 0.62$ & $2.29 \pm 0.36$ & 0.7042 \\
\hline & First dose (day 1) & $5.41 \pm 0.60$ & $5.36 \pm 0.91$ & $6.10 \pm 1.03$ & $5.91 \pm 0.85$ & $5.54 \pm 0.68$ & 0.0898 \\
\hline & Baseline-adjusted & $3.13 \pm 0.53$ & $2.99 \pm 1.03$ & $3.96 \pm 0.90$ & $3.57 \pm 1.00$ & $3.26 \pm 0.88$ & 0.0432 \\
\hline & Last dose (day 5) & $5.59 \pm 0.71$ & $5.73 \pm 0.88$ & $6.09 \pm 0.62$ & $6.22 \pm 0.47$ & $6.15 \pm 0.59$ & 0.0609 \\
\hline & Baseline-adjusted & $3.38 \pm 0.65$ & $3.36 \pm 0.89$ & $3.93 \pm 1.00$ & $3.88 \pm 0.69$ & $3.86 \pm 0.74$ & 0.1649 \\
\hline \multirow[t]{5}{*}{$12-24 \mathrm{~h}$} & Baseline & $1.83 \pm 0.62$ & $1.81 \pm 0.49$ & $1.87 \pm 0.35$ & $2.06 \pm 0.97$ & $1.76 \pm 0.37$ & 0.7342 \\
\hline & First dose (day 1) & $4.04 \pm 1.12$ & $5.69 \pm 0.84$ & $4.45 \pm 1.53$ & $6.09 \pm 0.89$ & $5.79 \pm 0.95$ & $<0.0001$ \\
\hline & Baseline-adjusted & $2.21 \pm 1.15$ & $3.87 \pm 0.74$ & $2.58 \pm 1.39$ & $4.04 \pm 1.13$ & $4.03 \pm 0.83$ & $<0.0001$ \\
\hline & Last dose (day 5) & $3.73 \pm 1.30$ & $5.64 \pm 0.80$ & $4.43 \pm 1.25$ & $6.07 \pm 0.57$ & $5.60 \pm 0.87$ & $<0.0001$ \\
\hline & Baseline-adjusted & $2.00 \pm 1.22$ & $3.83 \pm 0.98$ & $2.55 \pm 1.39$ & $3.98 \pm 1.14$ & $3.84 \pm 0.77$ & $<0.0001$ \\
\hline \multirow[t]{3}{*}{$0-4 \mathrm{~h}$} & Baseline & $2.13 \pm 0.38$ & $1.81 \pm 0.32$ & $2.20 \pm 0.75$ & $2.11 \pm 0.62$ & $2.06 \pm 0.31$ & 0.3324 \\
\hline & First dose (day 1) & $4.12 \pm 1.25$ & $4.32 \pm 1.16$ & $5.46 \pm 1.37$ & $5.21 \pm 1.36$ & $4.16 \pm 0.80$ & 0.0082 \\
\hline & Baseline-adjusted & $1.98 \pm 1.13$ & $2.51 \pm 1.21$ & $3.26 \pm 1.15$ & $3.10 \pm 1.32$ & $2.11 \pm 0.84$ & 0.0126 \\
\hline
\end{tabular}

$p$ values represent the comparison among the five groups

$S D$ standard deviation, $q d$ once daily, $q 12 h$ every $12 \mathrm{~h}$

Table 6 Change in percentage time with the intragastric $\mathrm{pH} \geq 4.0$ after single or repeated intravenous administration of dexlansoprazole 15 or $30 \mathrm{mg}$ and lansoprazole $30 \mathrm{mg}(\mathrm{mean} \pm \mathrm{SD})$

\begin{tabular}{|c|c|c|c|c|c|c|c|}
\hline \multirow{2}{*}{ Time period } & \multirow{2}{*}{ Variable } & \multicolumn{4}{|c|}{ Dexlansoprazole group } & \multirow{2}{*}{$\begin{array}{l}\text { Lansoprazole group } \\
\text { E (30 mg q12h) }\end{array}$} & \multirow{2}{*}{$p$ value } \\
\hline & & A (15 mg qd) & B (15 mg q12h) & $\mathrm{C}(30 \mathrm{mg} \mathrm{qd})$ & $\mathrm{D}(30 \mathrm{mg} \mathrm{q} 12 \mathrm{~h})$ & & \\
\hline \multirow[t]{5}{*}{$0-24 \mathrm{~h}$} & Baseline & $10.12 \pm 7.30$ & $11.21 \pm 6.75$ & $8.47 \pm 5.66$ & $11.46 \pm 13.18$ & $8.56 \pm 5.85$ & 0.8000 \\
\hline & First dose (day 1) & $61.14 \pm 16.32$ & $76.31 \pm 11.98$ & $67.34 \pm 20.92$ & $86.17 \pm 11.52$ & $79.69 \pm 15.30$ & 0.0007 \\
\hline & Baseline-adjusted & $51.02 \pm 15.02$ & $65.11 \pm 14.50$ & $58.86 \pm 19.23$ & $74.71 \pm 13.33$ & $71.13 \pm 18.49$ & 0.0025 \\
\hline & Last dose (day 5) & $61.45 \pm 18.92$ & $83.58 \pm 12.34$ & $70.14 \pm 15.65$ & $93.39 \pm 5.97$ & $87.26 \pm 12.08$ & $<0.0001$ \\
\hline & Baseline-adjusted & $53.04 \pm 16.81$ & $72.37 \pm 15.15$ & $61.38 \pm 19.20$ & $81.44 \pm 13.83$ & $78.70 \pm 12.77$ & $<0.0001$ \\
\hline \multirow[t]{5}{*}{$0-12 \mathrm{~h}$} & Baseline & $14.05 \pm 8.98$ & $15.34 \pm 7.65$ & $11.84 \pm 7.48$ & $12.89 \pm 11.12$ & $13.21 \pm 9.04$ & 0.8765 \\
\hline & First dose (day 1) & $73.47 \pm 9.63$ & $71.26 \pm 15.79$ & $80.52 \pm 15.92$ & $82.36 \pm 13.14$ & $73.81 \pm 12.68$ & 0.1505 \\
\hline & Baseline-adjusted & $59.42 \pm 7.78$ & $55.92 \pm 19.67$ & $68.68 \pm 13.78$ & $69.47 \pm 17.58$ & $60.60 \pm 19.25$ & 0.1279 \\
\hline & Last dose (day 5) & $79.22 \pm 12.52$ & $83.01 \pm 13.23$ & $85.37 \pm 11.12$ & $92.85 \pm 7.29$ & $89.92 \pm 10.01$ & 0.0172 \\
\hline & Baseline-adjusted & $66.68 \pm 14.88$ & $67.66 \pm 14.97$ & $73.04 \pm 16.66$ & $79.72 \pm 11.25$ & $76.71 \pm 15.85$ & 0.1185 \\
\hline \multirow[t]{5}{*}{$12-24 \mathrm{~h}$} & Baseline & $6.20 \pm 9.89$ & $7.05 \pm 9.79$ & $5.09 \pm 4.76$ & $10.51 \pm 17.33$ & $3.91 \pm 6.17$ & 0.5358 \\
\hline & First dose (day 1) & $48.70 \pm 24.85$ & $81.31 \pm 13.74$ & $54.09 \pm 29.86$ & $89.96 \pm 11.57$ & $85.54 \pm 20.04$ & $<0.0001$ \\
\hline & Baseline-adjusted & $42.50 \pm 25.45$ & $74.26 \pm 14.49$ & $48.99 \pm 28.26$ & $79.46 \pm 15.49$ & $81.62 \pm 20.17$ & $<0.0001$ \\
\hline & Last dose (day 5) & $43.64 \pm 29.67$ & $84.08 \pm 13.58$ & $54.85 \pm 22.99$ & $93.88 \pm 6.15$ & $84.54 \pm 17.03$ & $<0.0001$ \\
\hline & Baseline-adjusted & $39.35 \pm 28.71$ & $77.03 \pm 18.26$ & $49.66 \pm 25.08$ & $82.58 \pm 18.13$ & $80.63 \pm 15.68$ & $<0.0001$ \\
\hline \multirow[t]{3}{*}{$0-4 \mathrm{~h}$} & Baseline & $12.55 \pm 6.21$ & $6.73 \pm 5.31$ & $12.48 \pm 11.25$ & $9.64 \pm 9.49$ & $8.71 \pm 6.35$ & 0.2698 \\
\hline & First dose (day 1) & $44.02 \pm 25.73$ & $49.81 \pm 25.18$ & $67.31 \pm 25.18$ & $68.33 \pm 26.25$ & $47.26 \pm 19.25$ & 0.0218 \\
\hline & Baseline-adjusted & $31.47 \pm 25.35$ & $43.08 \pm 26.00$ & $54.84 \pm 20.98$ & $58.69 \pm 26.23$ & $38.54 \pm 21.90$ & 0.0254 \\
\hline
\end{tabular}

$p$ values represent the comparison among the five groups

$S D$ standard deviation, $q d$ once daily, $q 12 h$ every $12 \mathrm{~h}$ 
Table 7 Change in percentage time with the intragastric $\mathrm{pH} \geq 6.0$ after single or repeated intravenous administration of dexlansoprazole 15 or $30 \mathrm{mg}$ and lansoprazole $30 \mathrm{mg}$ (mean $\pm \mathrm{SD})$

\begin{tabular}{|c|c|c|c|c|c|c|c|}
\hline \multirow[t]{2}{*}{ Time period } & \multirow[t]{2}{*}{ Variable } & \multicolumn{4}{|c|}{ Dexlansoprazole group } & \multirow{2}{*}{$\begin{array}{l}\text { Lansoprazole group } \\
\text { E (30 mg q12h) }\end{array}$} & \multirow[t]{2}{*}{$p$ value } \\
\hline & & A (15 mg qd) & B (15 mg q12h) & $\mathrm{C}(30 \mathrm{mg} \mathrm{qd})$ & D (30 mg q12h) & & \\
\hline \multirow[t]{5}{*}{$0-24 \mathrm{~h}$} & Baseline & $3.36 \pm 4.92$ & $2.89 \pm 2.73$ & $2.54 \pm 2.73$ & $3.20 \pm 4.39$ & $2.60 \pm 2.86$ & 0.9684 \\
\hline & First dose (day 1) & $36.52 \pm 14.72$ & $49.81 \pm 17.29$ & $48.56 \pm 23.21$ & $58.89 \pm 20.43$ & $51.99 \pm 16.30$ & 0.0496 \\
\hline & Baseline-adjusted & $33.16 \pm 14.22$ & $46.93 \pm 17.15$ & $46.03 \pm 21.98$ & $55.69 \pm 18.65$ & $49.39 \pm 16.31$ & 0.0320 \\
\hline & Last dose (day 5) & $35.70 \pm 16.17$ & $53.70 \pm 17.06$ & $49.99 \pm 17.92$ & $62.62 \pm 15.32$ & $57.00 \pm 16.35$ & 0.0013 \\
\hline & Baseline-adjusted & $33.45 \pm 13.85$ & $50.81 \pm 16.63$ & $47.38 \pm 19.34$ & $59.20 \pm 15.62$ & $54.40 \pm 15.61$ & 0.0023 \\
\hline \multirow[t]{5}{*}{$0-12 \mathrm{~h}$} & Baseline & $2.58 \pm 2.61$ & $3.62 \pm 2.65$ & $3.15 \pm 3.73$ & $2.71 \pm 3.38$ & $2.80 \pm 3.44$ & 0.9169 \\
\hline & First dose (day 1) & $51.90 \pm 15.68$ & $49.68 \pm 19.74$ & $65.66 \pm 22.02$ & $59.89 \pm 19.83$ & $53.36 \pm 12.95$ & 0.1458 \\
\hline & Baseline-adjusted & $49.32 \pm 15.12$ & $46.06 \pm 21.03$ & $62.51 \pm 20.99$ & $57.18 \pm 20.52$ & $50.56 \pm 14.42$ & 0.1512 \\
\hline & Last dose (day 5) & $53.06 \pm 17.16$ & $56.18 \pm 21.18$ & $68.25 \pm 14.36$ & $68.85 \pm 12.69$ & $66.26 \pm 15.26$ & 0.0370 \\
\hline & Baseline-adjusted & $51.29 \pm 15.74$ & $52.56 \pm 21.09$ & $64.91 \pm 16.76$ & $66.00 \pm 11.22$ & $63.46 \pm 15.95$ & 0.0538 \\
\hline \multirow[t]{5}{*}{$12-24 \mathrm{~h}$} & Baseline & $4.12 \pm 8.42$ & $2.12 \pm 4.15$ & $1.93 \pm 3.03$ & $4.17 \pm 7.60$ & $2.42 \pm 4.85$ & 0.7534 \\
\hline & First dose (day 1) & $21.14 \pm 15.27$ & $49.96 \pm 21.75$ & $31.42 \pm 28.10$ & $57.83 \pm 26.22$ & $50.61 \pm 23.24$ & 0.0005 \\
\hline & Baseline-adjusted & $17.02 \pm 16.61$ & $47.84 \pm 20.09$ & $29.49 \pm 26.65$ & $53.66 \pm 24.38$ & $48.19 \pm 21.71$ & 0.0002 \\
\hline & Last dose (day 5) & $18.35 \pm 19.05$ & $51.18 \pm 19.24$ & $31.68 \pm 23.87$ & $56.35 \pm 19.57$ & $47.74 \pm 20.07$ & $<0.0001$ \\
\hline & Baseline-adjusted & $15.63 \pm 17.83$ & $49.06 \pm 18.36$ & $29.82 \pm 24.46$ & $51.86 \pm 21.38$ & $45.31 \pm 18.53$ & $<0.0001$ \\
\hline \multirow[t]{3}{*}{$0-4 \mathrm{~h}$} & Baseline & $2.90 \pm 3.54$ & $2.88 \pm 3.73$ & $5.39 \pm 7.00$ & $3.55 \pm 4.78$ & $2.69 \pm 3.31$ & 0.5385 \\
\hline & First dose (day 1) & $28.15 \pm 24.64$ & $30.44 \pm 19.61$ & $52.01 \pm 28.29$ & $47.79 \pm 28.82$ & $24.52 \pm 16.42$ & 0.0089 \\
\hline & Baseline-adjusted & $25.25 \pm 24.14$ & $27.56 \pm 20.22$ & $46.62 \pm 25.16$ & $44.24 \pm 28.28$ & $21.84 \pm 18.30$ & 0.0158 \\
\hline
\end{tabular}

$p$ values represent the comparison among the five groups

$S D$ standard deviation, $q d$ once daily, $q 12 h$ every $12 \mathrm{~h}$

B) or $30 \mathrm{mg}$ (group D), or lansoprazole $30 \mathrm{mg}$ (group E), was $5.73 \pm 0.88,6.22 \pm 0.47$, and $6.15 \pm 0.59$, respectively. The median intragastric $\mathrm{pH}, \mathrm{TpH} \geq 4.0(\%)$ and $\mathrm{TpH} \geq$ $6.0(\%) 0-12 \mathrm{~h}$ after the last morning dose in group $\mathrm{B}$ was $6.11 \pm 0.78,83.01 \pm 13.23$ and $56.18 \pm 21.18$, respectively, which were similar to those in group E, i.e. $6.54 \pm 0.56$, $89.92 \pm 10.01$ and $66.26 \pm 15.26$, respectively. The median intragastric $\mathrm{pH}, \mathrm{TpH} \geq 4.0(\%)$ and $\mathrm{TpH} \geq 6.0(\%)$ in group $\mathrm{D}$ was $6.50 \pm 0.33,92.85 \pm 7.29$ and $68.85 \pm 12.69$, respectively. Zero to $24 \mathrm{~h}$ after the last morning dose, median and mean intragastric $\mathrm{pH}, \mathrm{TpH} \geq 4.0(\%)$ and $\mathrm{TpH} \geq 6.0(\%)$ in group B were $6.07 \pm 0.61,5.70 \pm 0.76,83.58 \pm 12.34$ and $53.70 \pm 17.06$, respectively, which were also similar to those in group E, i.e. $6.15 \pm 0.62,5.88 \pm 0.67,87.26 \pm 12.08$ and $57.00 \pm 16.35$, respectively. An increment was found in the median and mean intragastric $\mathrm{pH}, \mathrm{TpH} \geq 4.0(\%)$ and $\mathrm{TpH} \geq$ $6.0(\%)$ in group D compared with group B, i.e. $6.34 \pm 0.45$, $6.15 \pm 0.50,93.39 \pm 5.97$ and $62.62 \pm 15.32$, respectively. However, no statistically significant difference of all the pharmacodynamic parameters was observed among these three groups.

No significant differences in baseline-adjusted median and mean intragastric $\mathrm{pH}$, baseline-adjusted $\mathrm{TpH} \geq 4.0(\%)$ and $\mathrm{TpH} \geq 6.0(\%)$ were observed between groups $\mathrm{B}$ and $\mathrm{E}$ during any intervals. Baseline-adjusted median and mean intragastric $\mathrm{pH}, \mathrm{TpH} \geq 4.0(\%)$ and $\mathrm{TpH} \geq 6.0(\%)$ for group
D were significantly higher than those of group B, but only $0-4 \mathrm{~h}$ after the first dose.

\subsection{Pharmacokinetic/Pharmacodynamic Relationship}

The pharmacokinetic/pharmacodynamic relationship between dexlansoprazole $\mathrm{AUC}_{\mathrm{t}}$ and baseline-adjusted $\mathrm{TpH} \geq 4.0(\%)$ or $\mathrm{TpH} \geq 6.0(\%) 0-12 \mathrm{~h}$ after a single dose was assessed by putting all the data from the dexlansoprazole groups together, and excluding the lansoprazole group. Three cases with an $\mathrm{AUC}_{\mathrm{t}}>15,000 \mathrm{ng} \cdot \mathrm{h} / \mathrm{mL}$ were excluded directly from the scatter diagram. As shown in Fig. 3, there was a moderate positive correlation between $\mathrm{AUC}_{\mathrm{t}}$ and baseline-adjusted $\mathrm{TpH} \geq 4.0(\%)$ or $\mathrm{TpH} \geq$ $6.0(\%)$ over $0-12 \mathrm{~h}$, with Pearson correlation coefficients of 0.604 and 0.480 , respectively $(p=0.000)$. After $\mathrm{q} 12 \mathrm{~h}$ multiple dosing, a weak positive correlation between dexlansoprazole $\mathrm{AUC}_{\mathrm{t}}$ and baseline-adjusted $\mathrm{TpH} \geq 4.0(\%)$ over 0-24 h was observed, as shown in Fig. 4, with a Pearson correlation coefficient of $0.437(p=0.029)$, while the correlation between $\mathrm{AUC}_{\mathrm{t}}$ and baseline-adjusted $\mathrm{TpH} \geq$ $6.0(\%)$ over $0-24 \mathrm{~h}$ was not evaluated as the $\mathrm{TpH} \geq 6.0(\%)$ data were non-normally distributed. 
(a)

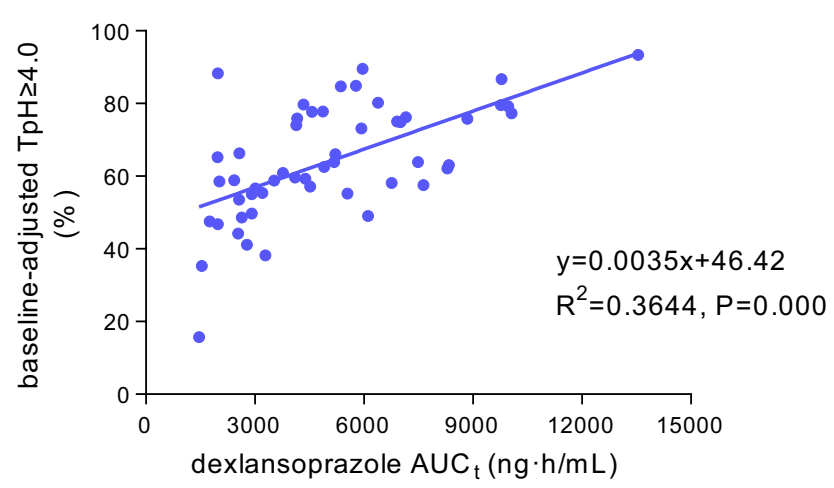

Fig. 3 Correlation of dexlansoprazole $\mathrm{AUC}_{\mathrm{t}}$ and baseline-adjusted a $\mathrm{TpH} \geq 4.0(\%)$ or b $\mathrm{TpH} \geq 6.0(\%) 0-12 \mathrm{~h}$ after a single administration. $A U C_{t}$ area under the concentration-time curve from time zero

\subsection{Safety and Tolerability}

A total of 31 AEs were recorded in 19 subjects during the study period, 19 (61.3\%) of which were identified through laboratory investigations. The vast majority of AEs (29/31, 93.5\%) were transient and considered mild (grade 1). One case of transient nasal pain as a grade $2 \mathrm{AE}$ was reported in group D (dexlansoprazole $30 \mathrm{mg} \mathrm{q} 12 \mathrm{~h}$ ), which was considered to be possibly related to $\mathrm{pH}$ catheter placement and was spontaneously recovered without intervention. Another subject in group C (dexlansoprazole $30 \mathrm{mg}$ qd) received 1 week of treatment with levofloxacin eye drops and systemic antibiotics due to conjunctivitis as a grade $2 \mathrm{AE}$, which was considered possibly not related to the drug because it happened prior to the administration of dexlansoprazole. The incidence of AEs is summarized in Table 8. No SAEs were reported. No participant was withdrawn due to AEs and no

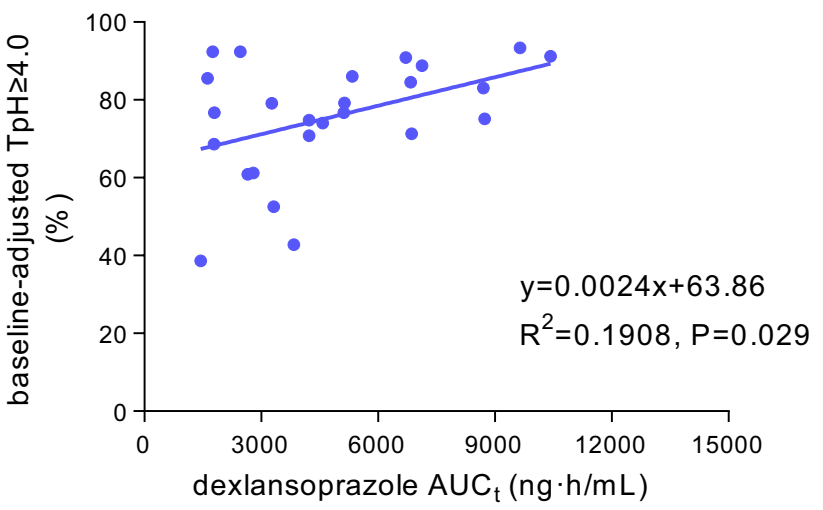

Fig. 4 Correlation of dexlansoprazole $\mathrm{AUC}_{\mathrm{t}}$ and baseline-adjusted $\mathrm{TpH} \geq 4.0(\%) 0-24 \mathrm{~h}$ after multiple dosing. $A U C_{t}$ area under the concentration-time curve from time zero to the last measurable concentration, $\mathrm{TpH} \geq 4.0$ (\%) percentage of time with the intragastric $\mathrm{pH}$ above 4.0

\section{(b)}

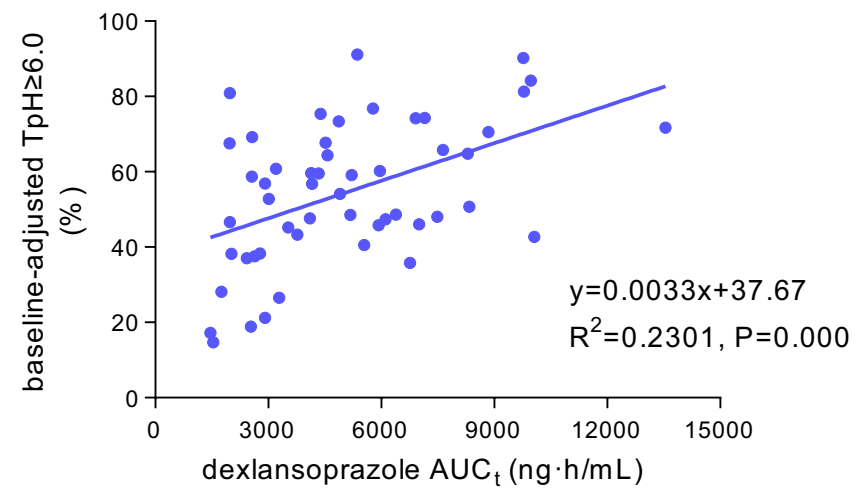

to the last measurable concentration, $T p H \geq 4.0(\%)$ percentage of time with the intragastric $\mathrm{pH}$ above 4.0, $T p H \geq 6.0(\%)$ percentage of time with the intragastric $\mathrm{pH}$ above 6.0

consistent, clinically significant changes from baseline were observed in vital signs, physical examinations, laboratory test results or ECG.

\section{Discussion}

To date, a few studies have been conducted providing the evidence for controlling intragastric $\mathrm{pH}$ after intravenous infusion of dexlansoprazole developed by other pharmaceutical companies, but the original injection preparation of lansoprazole has never been used in these studies, and no clearly improved acid inhibition profile was noted [11, 12].

In this study, we examined the pharmacokinetic and pharmacodynamic characteristics of the dexlansoprazole injection in the context of single and multiple administration, compared with the original lansoprazole injection, and confirmed that the dexlansoprazole injection was safe and well-tolerated during continuous dosing, with AEs of low incidence and mild severity.

Freston et al. reported that the $C_{\max }$ and AUC of dexlansoprazole were approximately $1200 \mathrm{ng} / \mathrm{mL}$ and $2500 \mathrm{ng} \cdot \mathrm{h} /$ $\mathrm{mL}$, respectively, following intravenous infusion of lansoprazole $30 \mathrm{mg}$ in Western healthy volunteers [7]. It is wellknown that a larger proportion of Asians have an impaired ability to metabolize PPIs than individuals of European and African descent, which would lead to higher PPI exposure (AUC and plasma levels) and more pronounced acid suppression effect in individuals of Asian ancestry [13]. In fact, at the same regimen, we found that both dexlansoprazole $C_{\max }$ and AUC were even higher than in Western subjects, with $C_{\max }$ and AUC being approximately $1800 \mathrm{ng} / \mathrm{mL}$ and $5800 \mathrm{ng} \cdot \mathrm{h} / \mathrm{mL}$, respectively. Consistent with these findings, results from our study showed that the $t_{1 / 2}$ and CL of dexlansoprazole were $1.76-2.06 \mathrm{~h}$ and $4.52-5.40 \mathrm{~L} / \mathrm{h}$, respectively, 
Table 8 Summary of study adverse events

\begin{tabular}{|c|c|c|c|c|c|}
\hline \multirow[t]{2}{*}{ Adverse event } & \multicolumn{4}{|c|}{ Dexlansoprazole group } & \multirow{2}{*}{$\begin{array}{l}\text { Lansoprazole group } \\
\text { E (30 mg q12h) }\end{array}$} \\
\hline & A (15 mg qd) & B (15 mg q12h) & $\mathrm{C}(30 \mathrm{mg}$ qd $)$ & $\mathrm{D}$ (30 mg q12h) & \\
\hline Subjects with at least one $\operatorname{AE}[n(\%)]$ & $3(21.43)$ & $5(35.71)$ & $3(21.43)$ & $5(35.71)$ & $3(21.43)$ \\
\hline Number of AEs & 6 & 8 & 3 & 10 & 4 \\
\hline Fecal occult blood & 1 & 3 & & 3 & 1 \\
\hline Urine-leukocytosis & & & & & 1 \\
\hline Elevated white blood cell count & & & & 2 & \\
\hline Elevated neutrophil count & & & & 1 & \\
\hline Leukopenia & 1 & & & & \\
\hline Neutropenia & 1 & & & & \\
\hline Decreased hemoglobin & & & & & 1 \\
\hline Elevated transaminase & & & & 1 & 1 \\
\hline Elevated uric acid & 1 & & & & \\
\hline Decreased fibrinogen & & 1 & & & \\
\hline Nasal pain & & & & 1 & \\
\hline Upper respiratory infection & & & 1 & & \\
\hline Conjunctivitis & & 1 & 1 & & \\
\hline Tendonitis & & 1 & & & \\
\hline Palpitation & 1 & & & & \\
\hline Fatigue & 1 & & & & \\
\hline Nausea & & 1 & 1 & & \\
\hline Chest discomfort & & 1 & & & \\
\hline Abdominal discomfort & & & & 1 & \\
\hline Dry cough & & & & 1 & \\
\hline
\end{tabular}

$A E$ adverse event, $q d$ once daily, $q 12 h$ every $12 \mathrm{~h}$

which were similar to the findings in the study by Li et al. [11], whereas the $t_{1 / 2}$ of dexlansoprazole was $1.17-1.21 \mathrm{~h}$ and CL was $6.62-6.80 \mathrm{~L} / \mathrm{h}$ in the study by Freston et al. The multiple dose pharmacokinetic parameters of $\mathrm{R}$ - or S-lansoprazole were similar to those observed following a single dose, indicating no accumulation after 5 days of $\mathrm{q} 12 \mathrm{~h}$ administration of dexlansoprazole or lansoprazole. Data from the lansoprazole $30 \mathrm{mg}$ group showed that the plasma concentrations of S-lansoprazole declined rapidly, with a $t_{1 / 2}$ of $0.87-1.02 \mathrm{~h}$ and a mean CL of approximately $35 \mathrm{~L} / \mathrm{h}$, which were consistent with those previous studies $[5,7,11]$.

Theoretically, lansoprazole, the racemic mixture, contains both the R- and S-enantiomer at the same equivalent, thus lansoprazole $30 \mathrm{mg}$ has a comparable R-enantiomer dose as dexlansoprazole $15 \mathrm{mg}$. However, in the present study, $\mathrm{AUC}_{\mathrm{t}}$ and $V_{\mathrm{d}}$ of dexlansoprazole were considerably higher in the lansoprazole $30 \mathrm{mg}$ group than in the dexlansoprazole $15 \mathrm{mg}$ group after either single or repeated administration, whereas the observed $C_{\max }$ of dexlansoprazole was similar. One possible reason for this finding is the limited number of individuals and the impact of interindividual variation due to metabolic phenotypes of $C Y P 2 C 19$, which is responsible for lansoprazole metabolism [5, 13].
Another possibility, i.e. the potential influence of S-lansoprazole in the delay of dexlansoprazole CL, has been proposed despite the fact that racemic conversion between dexlansoprazole and S-lansoprazole did not occur.

In pharmacodynamic evaluation, all pharmacodynamic parameters showed an obvious increase from baseline across all groups, indicating the definite gastric acid suppression achieved by administration of dexlansoprazole or lansoprazole. We did not design the lansoprazole oncedaily dosing group, and, on the first dosing day, individuals in the lansoprazole $30 \mathrm{mg} \mathrm{q} 12 \mathrm{~h}$ group were administered the second dose just $12 \mathrm{~h}$ after the first dose. Therefore, intragastric $\mathrm{pH}$ levels over $0-24 \mathrm{~h}$ in such different conditions as lansoprazole cannot be compared with those in a single-dose situation. A single dose of dexlansoprazole 30 $\mathrm{mg}$ resulted in a mean $24-\mathrm{h}$ intragastric $\mathrm{pH}$ of $5.28 \pm 1.15$, $24-\mathrm{h} \mathrm{TpH} \geq 4.0(\%)$ of $67.34 \pm 12.34$, and $24-\mathrm{h} \mathrm{TpH} \geq$ $6.0(\%)$ of $53.70 \pm 17.06$. These values were slightly lower than those reported by $\mathrm{Li}$ et al. partly due to the different manufacturers of dexlansoprazole. However, 0-24 h after $\mathrm{q} 12 \mathrm{~h}$ multiple dosing, mean intragastric $\mathrm{pH}$ for dexlansoprazole $30 \mathrm{mg}$ was the highest $(6.15 \pm 0.62)$, followed by lansoprazole $30 \mathrm{mg}(5.88 \pm 0.67)$ and dexlansoprazole 
$(5.70 \pm 0.76)$. These findings were similar with the previous report by $\mathrm{Li}$ et al. [11].

Increasing the dose frequency would improve acid suppression. As presumed, baseline-adjusted median and mean intragastric $\mathrm{pH}, \mathrm{TpH} \geq 4.0(\%)$ and $\mathrm{TpH} \geq 6.0(\%)$ for the $\mathrm{q} 12 \mathrm{~h}$ groups were significantly higher than those of the once-daily groups. However, baseline-adjusted median and mean intragastric $\mathrm{pH}, \mathrm{TpH} \geq 4.0(\%)$ and $\mathrm{TpH} \geq 6.0(\%)$ for the dexlansoprazole $30 \mathrm{mg}$ group were significantly higher than those of the $15 \mathrm{mg}$ group, but only $0-4 \mathrm{~h}$ after the first dose, suggesting that increasing the dose presented a transient better $\mathrm{pH}$ inhibition and had virtually little influence on whole-day gastric pH control. Although a statistically significant difference in dexlansoprazole $\mathrm{AUC}_{\mathrm{t}}$ was noted between lansoprazole $30 \mathrm{mg}$ and dexlansoprazole $15 \mathrm{mg}$, no significant differences in baseline-adjusted median and mean intragastric $\mathrm{pH}, \mathrm{TpH} \geq 4.0(\%)$ and $\mathrm{TpH} \geq 6.0(\%)$ were observed between dexlansoprazole $15 \mathrm{mg}$ and lansoprazole $30 \mathrm{mg}$ during any intervals. It was proposed that the persistent inhibition effect of dexlansoprazole beyond its short plasma $t_{1 / 2}$ [14] contributed to decreasing the differences in pharmacodynamic parameters between dexlansoprazole $15 \mathrm{mg}$ and lansoprazole $30 \mathrm{mg}$ in the context of repeated administration as enhanced gastric acid inhibition was achieved after 5 days of q12h dosing in both groups. Other possible causes, such as individual variations in proton pump turnover and saturation of covalent binding to the proton pump [14], should also be considered.

In addition, it is well-known that the degree of acid suppression was closely related to the total dose and the AUC $[14,15]$. In our pharmacokinetic/pharmacodynamic relationship study, a positive correlation between $\mathrm{AUC}_{\mathrm{t}}$ and baseline-adjusted $\mathrm{TpH} \geq 4.0(\%) 0-12 \mathrm{~h}$ after the first dose or 0-24 $\mathrm{h}$ after the last morning dose was noted, whereas there was no obvious correlation between $\mathrm{AUC}_{\mathrm{t}}$ and baselineadjusted $\mathrm{TpH} \geq 6.0(\%) 0-24 \mathrm{~h}$ after multiple dosing. This may be partly due to the full degree of gastric acid inhibition after multiple dosing of dexlansoprazole $15-30 \mathrm{mg}$, with $\mathrm{TpH} \geq 4.0(\%)$ and $\mathrm{TpH} \geq 6.0(\%)$ over $0-24 \mathrm{~h}$ being approximately $84-93 \%$ and $54-63 \%$.

Limitations of our research are that the number of participants was limited and genotyping of CYP2C19 was not performed prior to enrollment. The genotype variability of CYP2C19 led to confusion in the difference in pharmacokinetic parameters between the dexlansoprazole and lansoprazole groups. In addition, given the different protein-binding characteristics between the isomers, it would be worthwhile to determine the pharmacokinetic characteristics using free dexlansoprazole or s-lansoprazole concentrations. Another possible limitation is that we cannot exclude the possibility that other factors may affect the pharmacokinetic and pharmacodynamic findings during the study period. Furthermore, healthy subjects may not exhibit the same therapeutic effect as patients with active bleeding. Furthermore, the pharmacokinetic and pharmacodynamic relationship is relatively weak, assuming a linear, direct concentration-effect relationship in our study. Other non-linear models, such as a mechanism-based pharmacokinetic/pharmacodynamic model [16], which incorporates aspects of the physiological regulation of gastric acid production, the effects of food on gastric acid, and the complex effects of dexlansoprazole on gastric acid production, should be explored in order to predict optimal dosing regimens. Nevertheless, on the basis of our findings, we suggest that $\mathrm{q} 12 \mathrm{~h}$ dosing of dexlansoprazole $15 \mathrm{mg}$ has a similar gastric acid suppression effect compared with $\mathrm{q} 12 \mathrm{~h}$ dosing of lansoprazole $30 \mathrm{mg}$, which provides a reliable basis to proceed with studies in patients with peptic ulcer bleeding disease or seriously disordered gastrointestinal function.

\section{Conclusion}

The present study has clearly demonstrated that twicedaily intravenous dosing of dexlansoprazole up to $30 \mathrm{mg}$ was safe and well-tolerated in healthy Chinese subjects. Every $12 \mathrm{~h}$ dosing of dexlansoprazole $15 \mathrm{mg}$ has the comparable effect of gastric acid inhibition as lansoprazole $30 \mathrm{mg}$ twice daily.

Acknowledgements The authors would like to thank all study participants, as well as the clinical investigators, nurses, and study coordinators, for their contribution to this study.

Author Contributions LW was the principal investigator and was responsible for the study design, data acquisition, and data analysis, as well as writing and revising the article. JL participated in conducting the study as a sub-investigator and contributed to manuscript drafting. YZhe, YZha and ML conducted the study as investigators. JS contributed to the study design and data analysis. GW and DL contributed to statistics analysis. YZha contributed to quality control throughout the study. All authors have approved the final version of the text and have agreed to be accountable for this work.

\section{Compliance with Ethical Standards}

Funding This study was funded by Zhejiang Provincial Natural Science Foundation of China (No. LY19H280011).

Ethical Approval and Consent to Participate This study was conducted in accordance with the ethical standards of the institutional research committee and with the 1964 Helsinki declaration and its later amendments or comparable ethical standards. Written informed consent was obtained from all individual participants included in the study. Subjects were covered under the commercial insurance provided by the sponsor. 
Conflicts of interest Lihua Wu, Jian Liu, Yunliang Zheng, You Zhai, Meihua Lin, Guolan Wu, Duo Lv and Jianzhong Shentu declare that they have no conflicts of interest.

Open Access This article is distributed under the terms of the Creative Commons Attribution-NonCommercial 4.0 International License (http://creativecommons.org/licenses/by-nc/4.0/), which permits any noncommercial use, distribution, and reproduction in any medium, provided you give appropriate credit to the original author(s) and the source, provide a link to the Creative Commons license, and indicate if changes were made.

\section{References}

1. Lau JY, Barkun A, Fan DM, Kuipers EJ, Yang YS, Chan FK. Challenges in the management of acute peptic ulcer bleeding. Lancet. 2013;381(9882):2033-43.

2. Gralnek IM, Dumonceau JM, Kuipers EJ, Lanas A, Sanders DS, Kurien M, et al. Diagnosis and management of nonvariceal upper gastrointestinal hemorrhage: European Society of Gastrointestinal Endoscopy (ESGE) Guideline. Endoscopy. 2015;47(10):a1-46.

3. Barkun AN, Cockeram AW, Plourde V, Fedorak RN. Review article: acid suppression in non-variceal acute upper gastrointestinal bleeding. Aliment Pharmacol Ther. 1999;13(12):1565-84.

4. Worden JC, Hanna KS. Optimizing proton pump inhibitor therapy for treatment of nonvariceal upper gastrointestinal bleeding. Am J Health Syst Pharm. 2017;74(3):109-16.

5. Miura M. Enantioselective disposition of lansoprazole and rabeprazole in human plasma. Yakugaku Zasshi. 2006;126(6):395-402.

6. Miura M, Tada H, Yasui-Furukori N, Uno T, Sugawara K, Tateishi $\mathrm{T}$, et al. Pharmacokinetic differences between the enantiomers of lansoprazole and its metabolite, 5-hydroxylansoprazole, in relation to CYP2C19 genotypes. Eur J Clin Pharmacol. 2004;60(9):623-8.

7. Freston JW, Pilmer BL, Chiu YL, Wang Q, Stolle JC, Griffin JS, et al. Evaluation of the pharmacokinetics and pharmacodynamics of intravenous lansoprazole. Aliment Pharmacol Ther. 2004;19(10):1111-22.
8. Andersson T, Weidolf L. Stereoselective disposition of proton pump inhibitors. Clin Drug Investig. 2008;28(5):263-79.

9. Oldfield EC 4th, Parekh PJ, Johnson DA. Dexlansoprazole: delayed-release orally disintegrating tablets for the treatment of heartburn associated with non-erosive gastroesophageal reflux disease and the maintenance of erosive esophagitis. Expert Rev Gastroenterol Hepatol. 2016;10(10):1083-9.

10. Sun L, Cao Y, Jiao H, Fang Y, Yang Z, Bian M, et al. Enantioselective determination of (R)- and (S)-lansoprazole in human plasma by chiral liquid chromatography with mass spectrometry and its application to a stereoselective pharmacokinetic study. J Sep Sci. 2015;38(21):3696-703.

11. Li YQ, Yan ZY, Zhang HW, Sun LN, Jiao HW, Wang MF, et al. Safety, tolerability, pharmacokinetics and pharmacodynamics of dexlansoprazole injection in healthy Chinese subjects. Eur J Clin Pharmacol. 2017;73(5):547-54.

12. Sun LN, Cao Y, Li YQ, Fang YQ, Zhang HW, Wang MF, et al. Impact of Gastric $\mathrm{H}(+) / \mathrm{K}(+)$-ATPase rs 2733743 on the Intragastric $\mathrm{pH}$-Values of Dexlansoprazole Injection in Chinese Subjects. Front Pharmacol. 2017;8:670.

13. El Rouby N, Lima JJ, Johnson JA. Proton pump inhibitors: from CYP2C19 pharmacogenetics to precision medicine. Expert Opin Drug Metab Toxicol. 2018;14(4):447-60.

14. Shin JM, Kim N. Pharmacokinetics and pharmacodynamics of the proton pump inhibitors. J Neurogastroenterol Motil. 2013;19(1):25-35.

15. Park S, Hyun YJ, Kim YR, Lee JH, Ryu S, Kim JM, et al. Effects of CYP2C19 Genetic Polymorphisms on PK/PD Responses of Omeprazole in Korean Healthy Volunteers. J Korean Med Sci. 2017;32(5):729-36.

16. Puchalski TA, Krzyzanski W, Blum RA, Jusko WJ. Pharmacodynamic modeling of lansoprazole using an indirect irreversible response model. J Clin Pharmacol. 2001;41(3):251-8. 